\title{
MACHINING OF BOROSILICATE GLASS BY ECDM PROCESS: COMPARISON OF MACHINING PERFORMANCE DURING DRILLING AND SINKING HOLES
}

\author{
Om Prakash Gupta, Vinod Yadava \\ Motilal Nehru National Institute of Technology, Allahabad, India \\ prakash.mnnit.me@gmail.com
}

\begin{abstract}
Borosilicate glass has been set in the class of important engineering materials because of its excellent properties such as lower thermal expansion coefficient and lower density than soda lime glass, chemically inert, electrically non-conducting, optical clarity. Its applications are found in electronics industries, space exploration, chemical industries, optics, and implantable medical devices. The machining of borosilicate glass for creation of different types of features is difficult because of its brittle, nonconducting, and chemical inert nature. Electro-Chemical Discharge Machining is successfully utilized for machining of Borosilicate Glass. This paper discusses the comparison of machining performances of Electro-Chemical Discharge Machining process in two configurations, namely Drilling-ECDM and Sinking-ECDM, during machining of Borosilicate Glass. Machining performance is measured in terms of Material Removal Rate, Average Overcut and Hole Taper.
\end{abstract}

Index Terms- Borosilicate Glass, Electrochemical Discharge Machining, Drilling, Sinking

\section{INTRODUCTION}

Modern manufacturing industries which are involve in production of components used in electronics devices, space vehicles, laboratory equipments, optical instruments, implantable medical devices, have an increasing interest in materials with improved mechanical properties. Borosilicate Glass is one of them. However, it is fall into the category of difficult to cut materials. Conventional machining processes and most versatile unconventional machining process i.e Electro-Discharge Machining (EDM) and Electro-Chemical Machining (ECM) cannot be used to create different type of features on Borosilicate Glass because of its brittle and nonconducting nature.

It has been reported that Electro-Chemical Discharge Machining (ECDM) process has high potential for machining of various non-conducting, hard and brittle material. It is hybrid machining process which combines the features of EDM and ECM processes. It has been successfully overcome the limitation of electrical conductivity of materials. In this process several mechanism such as thermal machining, chemical machining, high temperature dissolution, micro cracking etc. may contribute to the material removal from workpiece. Beside all these potential mechanism available so far, the most acceptable mechanism of material removal is thermal machining and chemical machining.

ECDM was presented for the first time by Kurafuji and Suda (1968) as, Electro-Chemical Discharge Drilling for micro holes in glass. It has been shown that, the heat produced during electrochemical discharge phenomenon is as much high so that temperature rises up to melting point, sometimes even up to vaporization temperature of the glass[1],[2]. ECDM process is not only limited to machining of glass, this process can be successfully used for machining of various nonconducting, hard and brittle materials such as ceramics, carbides, composite, quartz etc [3],[4],[5]. ECDM process can be used in four different configurations such as DrillingECDM, Sinking-ECDM, Milling-ECDM and Travelling wireECDM. In ECDM process electrical discharge takes place at smaller electrode called as tool electrode, generally cathode is made smaller then anode. However, both electrodes can be simultaneously used as tool. Anode can also be made smaller; electrical discharge can also achieve at anode [3]. The applied voltage between electrodes and specific conductance of electrolyte has greater impact on MRR, TWR and Overcut during ECDM process [6]. It was reported that during machining of Kevlar-fiber-epoxy and glass-fiber-epoxy composites, MRR, TWR and Overcut increase due to increase in applied voltage between electrodes and increase in specific conductance of electrolyte. In case of Kevlar-fiber-epoxy composite, MRR, TWR and radial Overcut were increases with increase in specific conductance and decreases with tool diameter [7]. In case of e-glass-fiber-epoxy composite maximum MRR is obtained around $70 \mathrm{~V} \mathrm{DC}$ voltage and 80 $\mathrm{g} / \mathrm{l}$ of electrolyte concentration, has been reported [8]. Initially there was constant DC power supply used as source but it has been proved that pulsated DC power source is more suitable for ECDM process. It was also reported that, the influential parameters i.e., applied voltage and duty factor of pulsated DC power supply improves the MRR [4]. Use of longitudinal vibration of the electrode tool does not have significant effect on the process while a rotating micro drill is used as electrode tool [9]. 
ECDM process is not only limited to fabrication of holes, moreover it has been incorporated for fabrication of micro channels, micro-texturing of surfaces too. ECDM process is suitable for fabrication of micro channels on glass. The depth of micro channels has been obtained in range of $75 \mu \mathrm{m}-120$ $\mu \mathrm{m}$ [10]. During surface texture of glass micro channels, it was observed, feathery like patterns were obtained for low electrolyte concentration. However, spongy and porous structure results with high electrolyte concentration [11].

Form literature review it has been concluded that, there was few investigations have been carried out on Borosilicate Glass as compared to soda lime glass. The present research work aims to the machining of Borosilicate Glass by ECDM process. In this paper, a comparison has been made between machining performances of drilling and sinking configurations of ECDM process.

\section{EXPERIMENTATION}

In the present research work, a self developed experimental setup of drilling/sinking ECDM process has been used for drilling and sinking holes on borosilicate glass. Figure 1 shows the photograph of the setup of Drilling/Sinking-ECDM process. Pulsated DC power source has been used. Supply voltage and pulse-off time was kept constant at $45 \mathrm{~V}$ and $30 \mu$ s respectively. The hole was drilled by using a tool electrode whose diameter taken as $400 \mu \mathrm{m}$. $\mathrm{NaOH}$ was used as electrolyte.

One Parameter at a Time (OPAT) approach has been used to investigate the effect of Electrolyte Concentration (C), and Pulse-On Time $\left(\mathrm{T}_{\text {on }}\right)$ on the machining performance parameters such as MRR, Average Overcut and Hole Taper. MRR was calculated by weight difference method by using a microbalance to accuracy of $0.0001 \mathrm{~g}$.

In order to investigate the effect of input variables on Average Overcut and Hole Taper, the fabricated hole was observed by using a Compound Digital Trinocular Optical Microscope under magnification of 5X. The diameters of holes were approximated by choosing 25 points on edge of digital image of the hole. The Average Overcut was calculated by calculating difference between average diameter of hole and diameter of tool electrode. The hole taper was calculated by using following formula.

$$
\theta=\tan ^{-1}\left\{\frac{D_{\text {sntrancz }}-D_{\text {sxit }}}{2 t}\right\}
$$

Here $D_{\text {entrance }}$ and $D_{\text {exit }}$ are the entrance and exit diameters of hole, $\mathrm{t}$ is the thickness of the workpiece sample.

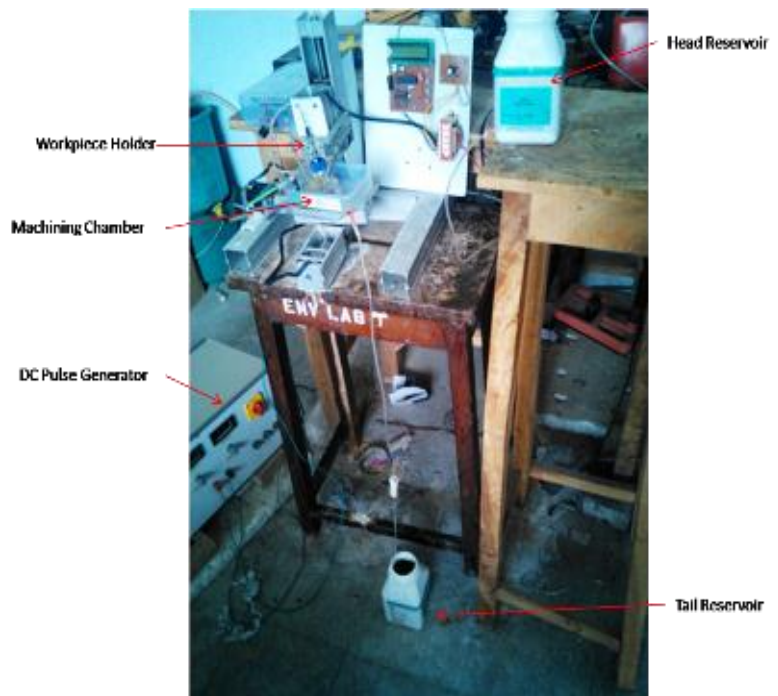

Figure 1. Photograph of the Setup of Drilling/Sinking-ECDM Process

\section{RESULTS AND DISCUSSION}

In the subsequent sections the effect of electrolyte concentration and pulse-on time has been discussed in terms of variation in MRR, Average Overcut and Hole Taper.

\subsection{Comparison of $M R R$}

The experiments were carried out to compare the MRR in drilling and sinking configurations of ECDM process, with respect to change in electrolyte concentration and pulse-on time. Experimental results are plotted on graph. Figure 2 shows the comparison of MRR in drilling and sinking configurations of ECDM process with change in electrolyte concentration during machining of Borosilicate Glass.

It is concluded from Fig.2; the MRR in drilling configuration is quite large as compared to sinking configuration of ECDM process. This behavior follows because; rotation of electrode tool improves the circulation of electrolyte solution, provides better flushing of debris, and ease of electrical discharge which reduces the machining time in drilling. Reduced machining time is responsible for large MRR in drilling as compared to sinking configuration of ECDM process. The calculated average MRR at electrolyte concentration of $150 \mathrm{~g} / \mathrm{l}$ is $8.3 \mathrm{mg} / \mathrm{min}$ and $0.75 \mathrm{mg} / \mathrm{min}$ for drilling and sinking configuration respectively. It is approx eleven times greater MRR; that is achieved in drilling than that of in sinking. 
ELK

Asia Pacific Journals

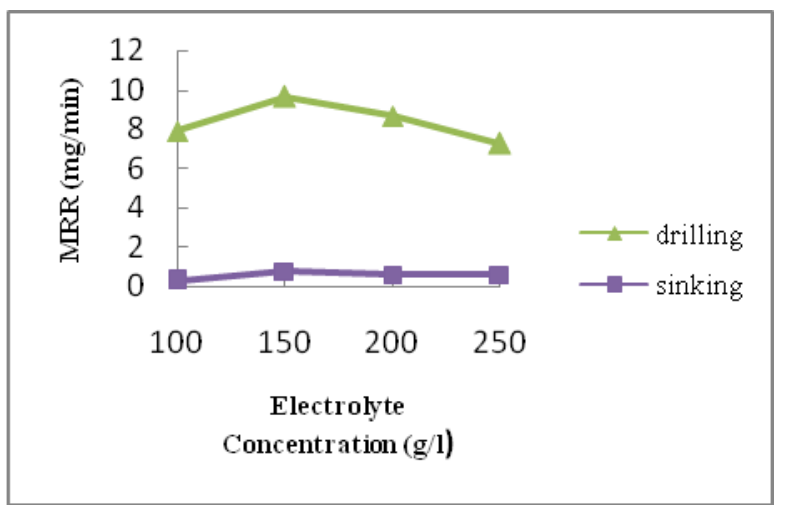

Figure 2. Comparison of MRR with Change in Electrolyte Concentration

Figure 2 also shows the variation of MRR with change in electrolyte concentration. It indicates that, MRR increases with increase in electrolyte concentration in range of $100 \mathrm{~g} / \mathrm{l}-$ $150 \mathrm{~g} / \mathrm{l}$, and shows decrease trend thereafter. This behavior follows the dependence on conductivity of solution with concentration of electrolyte. The conductivity of water- $\mathrm{NaOH}$ solution is increases with increase in concentration of $\mathrm{NaOH}$; the rate of electrochemical reaction and circuit current increases with increase in conductivity of electrolyte. The increased rate of electrochemical reaction results in more bubble generation, consequently probability of electrochemical discharge enhanced for given other input variables at constant value. Moreover, increased circuit current shows the heating effect, as developed heat is proportional to the circuit current, this results in enhanced rate of etching on the workpiece. Thus MRR increases; it is higher in electrolyte concentration around $150 \mathrm{~g} / \mathrm{l}$. As the concentration of $\mathrm{NaOH}$ increases beyond $150 \mathrm{~g} / \mathrm{l}$, movement of the ions is impede by collisions. In this way conductivity of electrolyte solution decreases, this is responsible for significantly increase in machining time. It was observed that material removal also increase, but MRR decrease due to higher machining time. A very high concentration of electrolyte solution is not suitable for higher MRR.

Figure 3 shows the comparison of MRR in drilling and sinking configurations of ECDM process with change in pulse-on time during machining of Borosilicate Glass. It is concluded from Fig. 3; that MRR is quite large in drilling as compared to sinking configuration of ECDM process. It is also concluded from Fig.2; in drilling, MRR increases with increase in pulse-on time. This behavior follows the availability of spark energy for longer time. An increase in pulse-on time implies more spark time at workpiece and hence higher value of MRR. However, the MRR in sinking
ELK Asia Pacific Journals - Special Issue

ISBN: 978-81-930411-8-5

configuration shows decrease trend after $45 \mu$ s of pulse-on time. This behavior follows because inadequacy of flushing impedes the removal of debris.

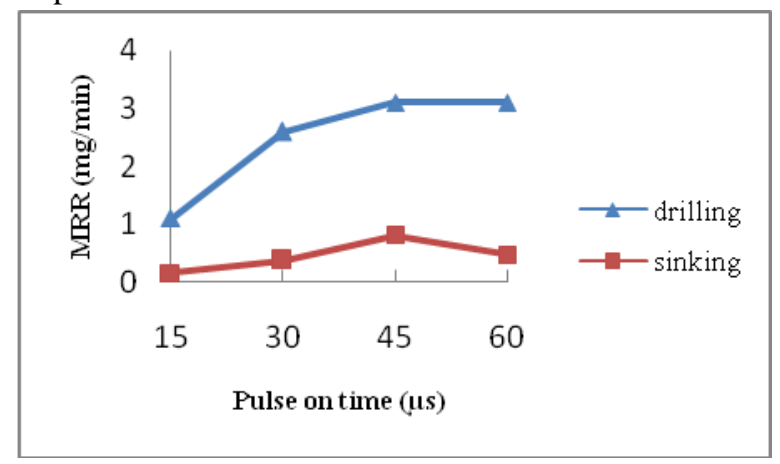

Figure 3. Comparison of MRR with Change in Pulse-On Time

It is concluded that rotation of electrode tool improves the MRR during machining of Borosilicate Glass.

\subsection{Comparison of Average Overcut}

The experiments were carried out to compare the average overcut in drilling and sinking configurations of ECDM process, with respect to change in electrolyte concentration and pulse-on time. Experimental results are plotted on graph. Figure 4 shows the comparison of average overcut in drilling and sinking configurations of ECDM process with change in electrolyte concentration during machining of Borosilicate Glass.

It is concluded from Fig. 4; the Average Overcut is more in sinking as compared to drilling configuration of ECDM process. This behavior follows because, lower MRR in sinking configuration increases the machining time, and hence lateral gap is enlarged due to side electrical discharge over a longer period of time.

The average overcut in sinking configuration is increases with increase in electrolyte concentration in range of $100 \mathrm{~g} / \mathrm{l}$ $150 \mathrm{~g} / \mathrm{l}$ and show decrease trend with further increase in electrolyte concentration. This can be understood on the basis of material removal in sinking. Since, the MRR first increases in electrolyte concentration range of $100 \mathrm{~g} / \mathrm{l}-150 \mathrm{~g} / \mathrm{l}$ and then decreases thereafter, so average overcut in sinking configuration shows such behavior. However, average overcut in drilling configuration increases with increase in electrolyte concentration. In higher electrolyte concentration machining time significantly increase, which is responsible for side enlargement of hole. 


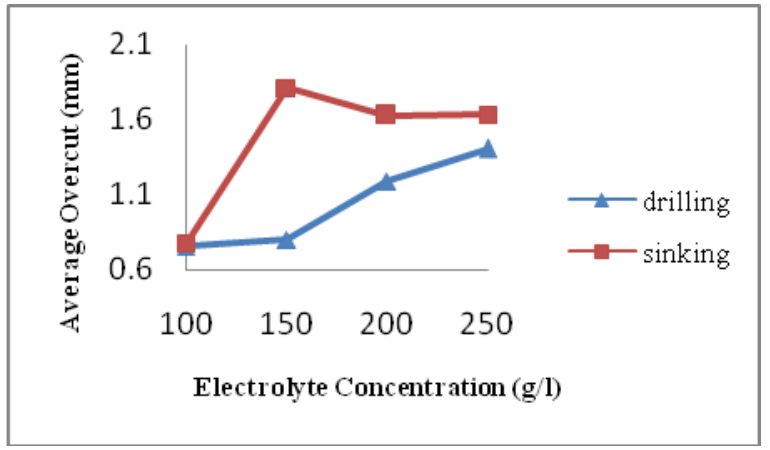

Figure 4. Comparison of Average Overcut with Change in Electrolyte Concentration

Figure 5 shows the comparison of average overcut in drilling and sinking configurations of ECDM process with change in pulse-on time. It is concluded that average overcut is more in sinking configuration as compared to drilling configuration. The difference in average overcut is very small in pulse-on time range of $15 \mu \mathrm{s}-45 \mu \mathrm{s}$, and increases with further increase in pulse-on time. Since, machining time for sinking is more than that of for drilling in pulse-on time range $45 \mu \mathrm{s}-65 \mu \mathrm{s}$, so side gap enlarged in sinking as compared to drilling. It was also observed that exit diameter is enlarged with increase in pulse-on time.

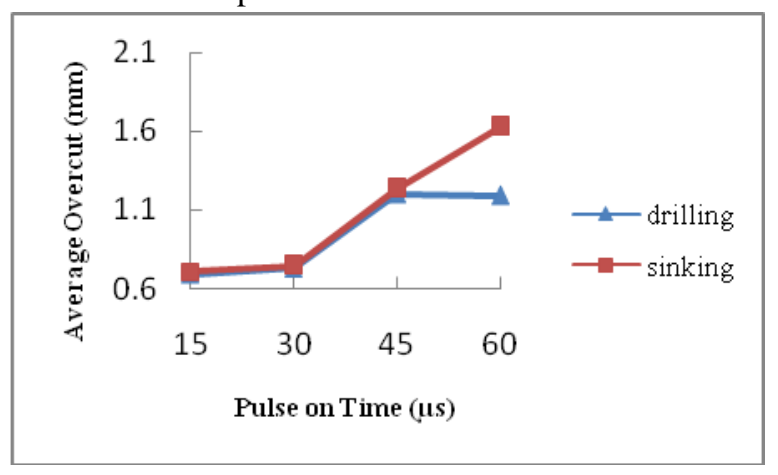

Figure 5. Comparison of Average Overcut with Change in Pulse-On Time

It is concluded that rotation of electrode tool reduces the average overcut during machining of Borosilicate Glass.

\subsection{Comparison of Hole Taper}

The experiments are carried out to compare the hole taper in drilling and sinking configurations of ECDM process, with respect to change in electrolyte concentration and Pulse-On Time. Experimental results are plotted on graph. Figure 6 shows the comparison of hole taper in drilling and sinking configurations of ECDM process with change in electrolyte concentration. It was observed that, hole taper is more in sinking as compared to drilling configuration of ECDM process. The graph shows, difference in hole taper is very small in electrolyte concentration range of $100 \mathrm{~g} / \mathrm{l}-150 \mathrm{~g} / \mathrm{l}$. However, there was significant difference was observed beyond electrolyte concentration of $150 \mathrm{~g} / \mathrm{l}$. This behavior follows because; entrance diameter during sinking is enlarged over a longer period of time due to insufficient flushing and inadequate circulation of electrolyte.

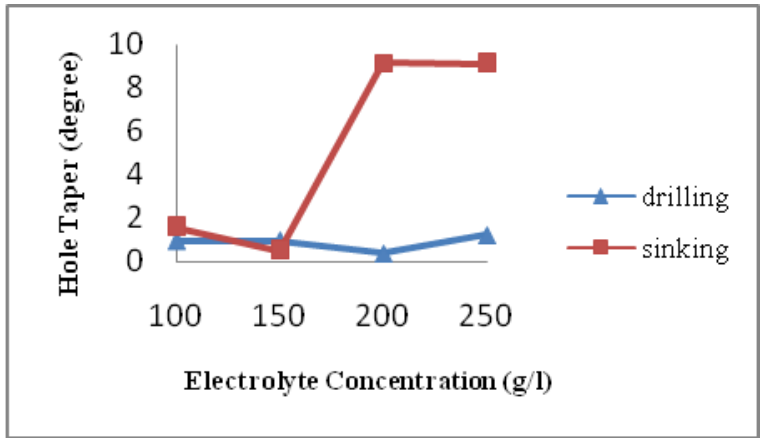

Figure 6. Comparison of Hole Taper with Change in Electrolyte Concentration

Figure 7 shows the comparison of hole taper in drilling and sinking configurations of ECDM process with change in pulse-on time. It is concluded from graph that, the hole is more taper in sinking as compared to drilling configuration of ECDM process. The difference in taper is increases with increase in pulse-on time. This behavior follows due to dependency of enlargement in entrance diameter on machining time. Figure 7 also shows that taper angle decreases with increase in pulse-on time. The hole taper decreases due to increase in exit diameter. This behavior follows because spark time and frontal discharge increase with increase in pulse-on time.

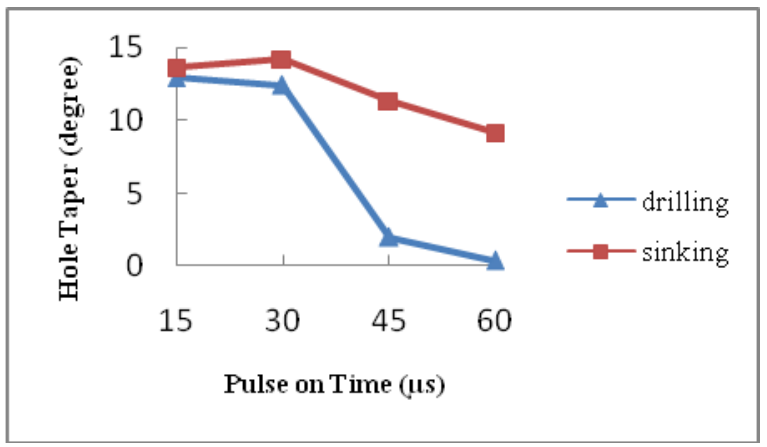

Figure 7. Comparison of Hole Taper with Change in Pulse-On Time 


\section{CONCLUSIONS}

In order to make holes on borosilicate glass, the machining performances of ECDM process has been compared in two configurations i.e. Drilling and Sinking configuration of ECDM process. It was concluded that MRR and accuracy of hole is better achieved in drilling as compared to sinking configuration of ECDM process.

\section{ACKNOWLEDGMENT}

Authors would like to thank Mechanical Engineering Department, MNNIT Allahabad, for providing the financial assistance for this experimental work of the project entitled "Development and Performance Study of Drilling/Sinking Electrochemical Spark Machining Process".

\section{REFERENCES}

[1] K. Allasu, A. Ghosh, M.K. Muju; "Preliminary qualitative approach of a proposed mechanism of material removal in electrical discharge machining of glass", European Journal of Mechanical Engineers, 36 (1992) 202-207

[2] A. Kulkarni, R. Sharan, G.K. Lal; "An experimental study of discharge mechanism in electrochemical discharge mechanism", International journal of machine tools manufacture, 42 (2002) 1121-1127

[3] B. Bhattacharya, B.N. Doloi, S.K. Sorkhel, "Experimental Investigation into Electrochemical Discharge Machining of non-conductive Ceramic Materials", Journal of Materials Processing Technology, 95 (1999) 145-154

[4] S.K. Chak, P.V. Rao, "Machining of SiC by ECDM Process using Different Electrode Configuration under the Effect of Pulsed DC", Int. J. Manufacturing Technology and Management, Vol. 28 Nos. 1/2/3, 2014

[5] J.W. Liu, T.M. Yue, Z.N. Guo; "An Analysis of Discharge Mechanism in Electrochemical Discharge Machining of Particulate Reinforced Metal Matrix Composites", Int. J. of Machine Tools and Manufacture, (86-96), 2010

[6] V.K. Jain, S.K. Chak, "Electrochemical Spark Trepanning of Alumina and Quartz", 4(2), 277-290, (2000)

[7] R. Wuthrich, V. Fascio, "Machining of non-conducting Materials using Electrochemical Discharge Phenomenon", Int. J. of Machine Tools and Manufacture, 45 (2005) 1095-1108

[8] S. Tandon, V.K. Jain, P. Kumar, K.P. Rajurkar, "Investigation into Machining of Composite", Precision Engineering

[9] A. Manna, V. Narang, "A Study on Micromachining of e-glass-fibreepoxy Composite be ECDM Process", Int. J. of Advanced manufacturing and Technology (2012) 61:1191-1197

[10] M.R. Razfar, J. Ni, "Study of the Effects of Tool Longitudinal Oscillation on the Machining Speed of Electrochemical Discharge Drilling of Glass", Precision Engineering 38 (2014) 885-892

[11] A.V. Kulkarni, V.K. Jain, K.A. Mishra, "Electrochemical Spark Micromachining of Metals and non-Metals", International Journal of Manufacturing Technology and Management, 22 (2), 107-123

[12] J.D.A. Ziki, T.F. Didar, R. Wuthrich, "Micro-Texturing Channel Surfaces on Glass with Spark Assisted Chemical Engraving", Int. J. of Machine Tools and Manufacture 57 (2012) 66-72 